\title{
Unisurgeon' uniportal video-assisted thoracoscopic surgery lobectomy
}

\author{
Diego Gonzalez-Rivas ${ }^{1,2}$ \\ ${ }^{1}$ Department of Thoracic Surgery and Minimally Invasive Thoracic Surgery Unit (UCTMI). Coruña University Hospital, Coruña, Spain; \\ ${ }^{2}$ Department of Thoracic Surgery, Shanghai Pulmonary Hospital, Tongji University School of Medicine, Shanghai 200433, China \\ Correspondence to: Diego Gonzalez-Rivas. Department of Thoracic Surgery, Coruña University Hospital, Xubias 84, Coruña 15006, Spain. \\ Email: diego.gonzalez.rivas@sergas.es.
}

\begin{abstract}
The video-assisted thoracoscopic surgery (VATS) for major pulmonary resections has evolved in a period of only 7 years from 3-4 incisions to a single incision approach. However, Uniportal VATS approach is different from other forms of minimally invasive thoracic surgery, and the technique of lung exposure and stapler insertion through a single hole should be learned step by step. The main advances of uniportal VATS during the last years are related to improvements in surgical technique, evolving to a concept of "advanced VATS instrumentation", and implementation of new technology. One recent advance in uniportal VATS is the possibility of using a robotic or pneumatic articulated arm that holds the camera stable and no needs a surgical assistant. This is called "unisurgeon uniportal VATS" in where the surgeon has more freedom of movements and eliminates the fatigue of assistant holding the camera. We are still in the beginning of the "unisurgeon era" that probably will be more popular in the next coming years thanks to the Implementation of wireless cameras and graspers by means of magnetic control.
\end{abstract}

Keywords: Unisurgeon; uniportal; robotic arm; minimally invasive surgery; future of surgery; lobectomy

Received: 12 September 2017; Accepted: 10 October 2017; Published: 07 November 2017.

doi: 10.21037/jovs.2017.10.07

View this article at: http://dx.doi.org/10.21037/jovs.2017.10.07

\section{Introduction}

Technical and medical progress derived from new ideas has produced tremendous benefits in the history of medicine and surgery. Essential to the advancement of thoracic surgery is the process of incorporating newer and latest technologies (1).

We as thoracic surgeons, always continue to learn new techniques and pursue the latest technology to improve our surgical skills to reduce morbidity and improve the health, wellness and survival of our patients. However in this way we normally find several obstacles; the high cost of new advanced technology, the difficult and slow acceptance by the traditional thinking and the hard learning curve at the beginning of using new technology.

The video-assisted thoracoscopic surgery (VATS) has evolved from 3-4 incisions to the uniportal VATS during the last years. The main advances of uniportal VATS during the last years are related to improvements in surgical technique and implementation of new technology (better staplers and energy devices, 3D and ultra high definition view) (2). The experience gained during the last years has allowed to modify the technique to develop tricks to easily manage the upper lobe vein and bronchus (both being the most difficult structures to divide), to use energy devices for hilar dissection (3) control most of the intraoperative bleedings (4) and perform the most complex procedures such as bronchovascular sleeve or carinal resections (5).

\section{Unisurgeon concept}

One of the latest advances in uniportal VATS is the possibility of using a robotic articulated arm that holds the camera stable. There are several available systems of articulated arms, such as one where the surgeon manually moves a pneumatic arm, and one where the arm is voicecontrolled. It is recommended to fix the articulated arm on 


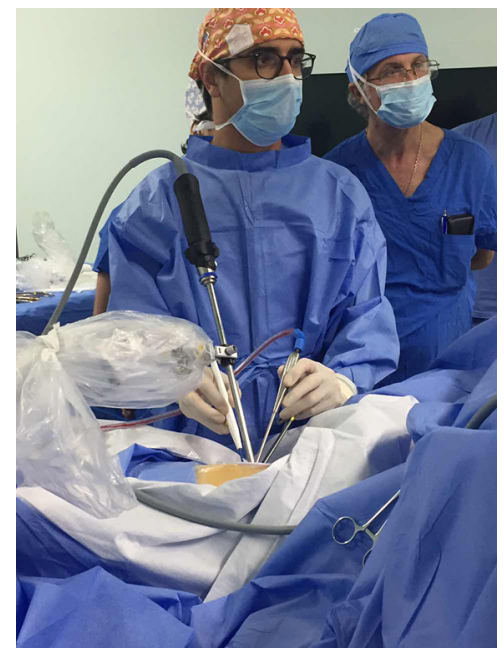

Figure 1 Bimanual instrumentation by the surgeon positioned in front of the patient.

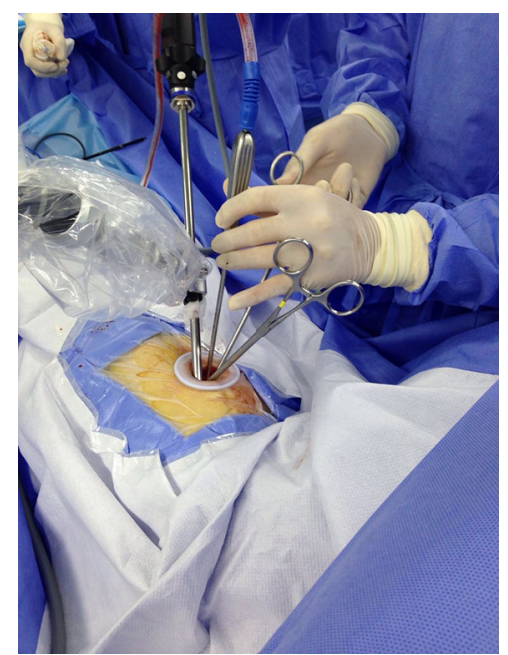

Figure 2 Double finger technique where the surgeon holds two instruments with the left hand.

the back position of the patient. This technology potentially obviates the need for an assistant altogether, and in the hands of very experienced uniportal VATS surgeons this enables a 'unisurgeon', assistant-free operation (Figure 1).

This approach requires that the surgeon must learn to handle several instruments with one hand and to be able to exert the same bimanual instrumentation. It is mandatory to learn "double finger instrumentation" which means that with one hand (usually the left hand) two instruments are held and moved at the same time: a retractor to grasp the lung and provide exposure with two digits, and a long

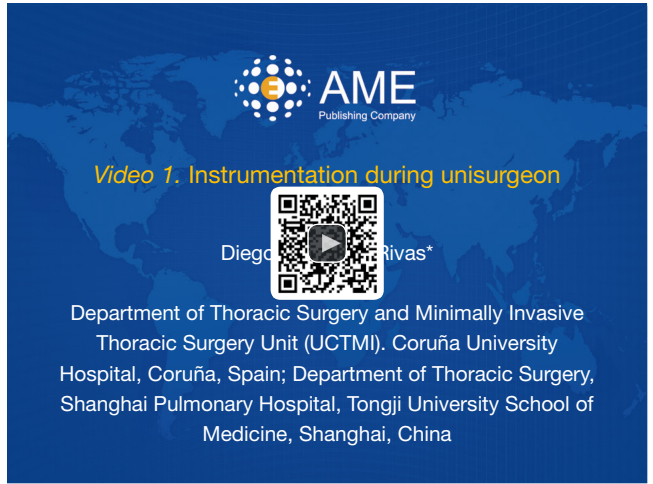

Figure 3 Instrumentation during unisurgeon procedure (6). Available online: http://www.asvide.com/articles/1770

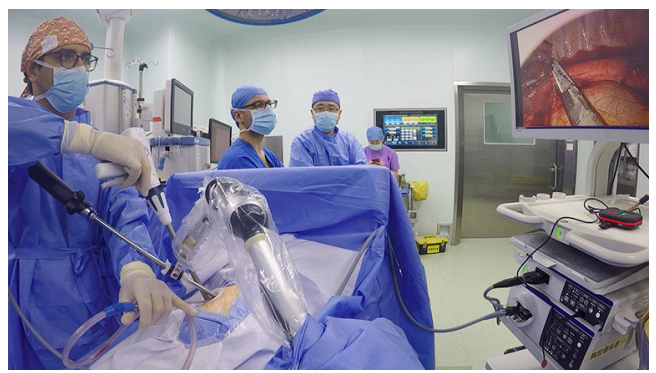

Figure 4 Division of inferior pulmonary vein during a left lower lobectomy.

curved suction to perform the tasks described above with the remaining digits (Figure 2). The right hand is used to wield the energy device or a dissector instrument as described above. Sometimes it is very helpful to support the ring clamp or grasper on a part of the articulated arm to release a hand to wield another instrument (Figure 3).

In conventional or Uniportal VATS, the camera is constantly moved by the assistant to actively maintain the zone of dissection in the middle of the screen. However, with the unisurgeon approach, it is the reverse: the camera is fixed and the surgeon must constantly adjust the retraction of the lung to position the dissection zone in the center of the screen. This is especially important for lobectomy, where we need to retract the lung cranially and caudally several times during the procedure (Figure 4). For segmental anatomic resections, where the area of dissection is more limited, we usually don't need to move the camera or the lung during the entire procedure (Figures 5 and 6).

There are some advantages to not having an assistant scrubbed in. First, an assistant often fatigues during an 


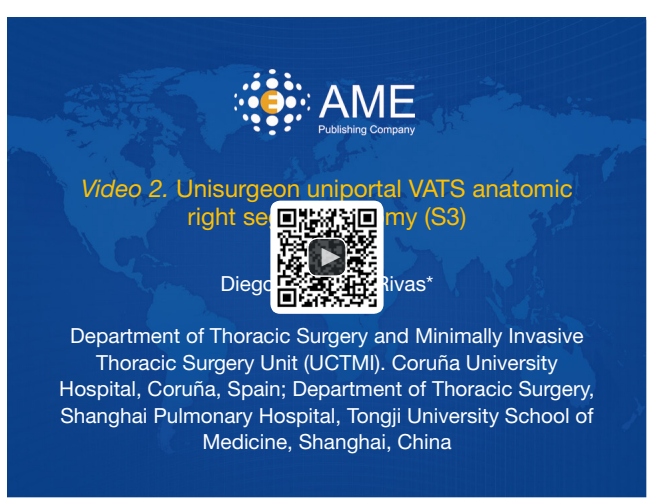

Figure 5 Unisurgeon uniportal VATS anatomic right segmentectomy (S3) (7). VATS, video-assisted thoracoscopic surgery. Available online: http://www.asvide.com/articles/1771

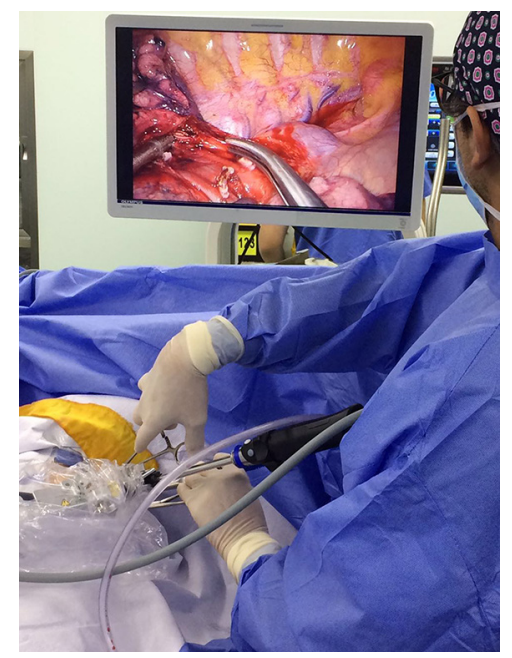

Figure 6 Upper lobe retraction and exposure to perform anatomic dissection during apical right segmentectomy (S1).

operation leading to worsening camera handling and retraction as the operation goes on. The unisurgeon approach may avoid this. Second, without an assistant standing right at the surgeon's elbow, the surgeon has more room to work comfortably on that side of the operating table, and a greater range of arm movements is possible without interference during the instrumentation. However, an assistant should always be available to be called in to help in case of bleeding or other on-table complications.

The adoption of the Unisurgeon technique is in line with modern era and technological advances. When the surgeon performs a uniportal approach, the role of the assistant is to hold the camera with one hand and a grasper to retract the lung with the other hand. The biggest development for Uniportal VATS surgery will come thanks to the wireless systems for cameras and graspers by means of magnetic control (graspers are already developed). Wireless cameras are still a prototype in the early period but they will include soon high definition and good mobility control. At this stage, surgeon will be able to reduce the size of incision and eliminate two elements through the wound, having more space to work. The assistant role will be just to move the camera and graspers by external magnetic fields allowing the surgeon to perform himself all the instrumentation in a very comfortable position.

Unisurgeon surgery is ideal for the surgeon when performing lower lobectomies (normally the assistant is located next to the surgeon), allowing more space and freedom of movements to the surgeon. In upper lobectomies the assistant holding the camera is normally placed on the patient's back so there is not much difference for the surgeon in terms of space for instrumentation. However, for lymph node dissection, the use of the robotic arm is very useful during lymphadenectomy at stations 2,4 , 5, 6 and 7 to allow the surgeon more space and freedom of movements for instrumentation.

\section{Discussion}

Uniportal VATS approach is distinct from other forms of minimally invasive thoracic surgery, and the technique of exposure and stapler insertion is totally different. Despite the learning curve for uniportal VATS being short, experience with conventional VATS alone is not enough to ensure total proficiency with uniportal VATS. Surgeons experienced with two ports technique or with anterior thoracotomy approach will probably adopt the uniportal approach faster than those experienced with three ports (8). However, the proper learning of crucial details and tricks of uniportal VATS technique requires visiting experienced high volume uniportal VATS centers, and attending training courses with hands-on wet labs run by the experts (9). Considering that we have only one hole and one direction, we must have in mind that precise movements are mandatory for an adequate exposure in uniportal VATS.

The uniportal technique has evolved during the last years to become almost an entirely new concept: "advanced uniportal VATS". This development emphasizes performing surgery with the least number of instruments possible, reducing the crowding of multiple instruments via the single intercostal space (9). Learning Uniportal thoracoscopic 
movements, with the use of an articulated or robotic arm, without the need for an assistant, opens new horizons of instrumentation, therefore facilitating surgeon's maneuvers. The Uniportal VATS is the ideal approach to encourage the industry to develop wireless systems and smaller surgical instruments that can be moved from the outside without having to remain through the incision. Likewise, the development of single incision robotic platforms will definitely change the future of thoracic surgery, helping the uniportal approach to become the standard technique in the next coming years. One thing is undeniable: we cannot stop the technological evolution in the surgical and anesthetic techniques because technology will definitely help the surgeon to be more accurate and accomplish the less invasive approach possible. In surgery as it happens in life, "less is more" (10).

\section{Acknowledgements}

None.

\section{Footnote}

Conflicts of Interest: The author has no conflicts of interest to declare.

Informed Consent: Written informed consent was obtained from the patient for publication of this manuscript and any accompanying images.

\section{References}

1. Gonzalez-Rivas D, Aymerich H, Eckland K. Balancing

doi: 10.21037/jovs.2017.10.07

Cite this article as: Gonzalez-Rivas D. Unisurgeon' uniportal video-assisted thoracoscopic surgery lobectomy. J Vis Surg 2017;3:163. the paradox of medical-surgical advances: a historical perspective on the current situation in thoracic surgery. $J$ Thorac Dis 2017;9:1168-70.

2. Ng CS, Lau KK, Gonzalez-Rivas D, et al. Evolution in surgical approach and techniques for lung cancer. Thorax 2013;68:681.

3. Gonzalez-Rivas D. Total anatomic vascular dissection for lobectomy by using only energy devices. J Vis Surg 2015;1:8.

4. Gonzalez-Rivas D, Stupnik T, Fernandez R, et al. Intraoperative bleeding control by uniportal video-assisted thoracoscopic surgery†. Eur J Cardiothorac Surg 2016;49 Suppl 1:i17-24.

5. Gonzalez-Rivas D, Yang Y, Stupnik T, et al. Uniportal video-assisted thoracoscopic bronchovascular, tracheal and carinal sleeve resections†. Eur J Cardiothorac Surg 2016;49 Suppl 1:i6-16.

6. Gonzalez-Rivas D. Instrumentation during unisurgeon procedure. Asvide 2017;4:453. Available online: http:// www.asvide.com/articles/1770

7. Gonzalez-Rivas D. Unisurgeon uniportal VATS anatomic right segmentectomy. Asvide 2017;4:454. Available online: http://www.asvide.com/articles/1771

8. Gonzalez-Rivas D, Paradela M, Fernandez R, et al. Uniportal video-assisted thoracoscopic lobectomy: two years of experience. Ann Thorac Surg 2013;95:426-32.

9. Gonzalez-Rivas D, Yang Y, Ng C. Advances in Uniportal Video-Assisted Thoracoscopic Surgery: Pushing the Envelope. Thorac Surg Clin 2016;26:187-201.

10. Gonzalez-Rivas D, Aymerich H, Bonome C, et al. From Open Operations to Nonintubated Uniportal VideoAssisted Thoracoscopic Lobectomy: Minimizing the Trauma to the Patient. Ann Thorac Surg 2015;100:2003-5. 